\title{
Validação de conteúdo de cenas do teste de conhecimento tático no tênis ${ }^{1}$
}

\author{
Scene content validation in a tactical \\ knowledge test of tennis
}

\author{
Layla Maria Campos ABURACHID \\ Pablo Juan GRECO²
}

\begin{abstract}
Resumo
Este estudo relata a utilização sistemática do método de validade de conteúdo usado para validar cenas de vídeo de jogos de tênis. Um painel de cinco peritos avaliou as imagens em movimento através dos critérios de clareza da linguagem, pertinência prática e representatividade do item. Os resultados ilustraram como cenas de jogos de tênis em situação de tomada de decisão podem se tornar itens validados, considerando-se valores acima de 0,80. A validação das cenas teve como objetivo estabelecer itens para a criação de um teste de conhecimento tático declarativo direcionado a avaliar o nível de tomada de decisão e percepção de tenistas. A utilização de cenas que apresentam imagens de situações reais do jogo de tênis, avaliadas por um painel de juízes, mostrou-se um procedimento eficaz para delimitação das cenas, com aproveitamento de 39 das 43 cenas selecionadas para a criação do teste.
\end{abstract}

Unitermos: Julgamento. Tênis. Validade de construto.

\begin{abstract}
This study reports on the systematic content validation method used to validate scenes in tennis video games. A panel of five experts evaluated the moving images through the criteria of clarity of language, practical relevance and the representativeness of the item. The results illustrate how scenes in tennis video games, in decision-making situations, can transform into validated items, considering values above 0.80 . The aim of the scene validation was to establish items to build a declarative, tactical knowledge test construct to evaluate tennis players'levels of decision making and perception. Bearing in mind that scenes represented real tennis situations, as evaluated by a panel of judges, it was shown to be an effective procedure for scene delimitation, with 39 of the 43 selected scenes being approved for the creation of the test.
\end{abstract}

Uniterms: Julgament. Tennis. Test validity.

A partir da década de 1980, tem-se buscado desenvolver trabalhos empíricos na área de estudos das capacidades táticas que oportunizam a melhoria dos processos de treinamento esportivo. A percepção e a tomada de decisão, assim como outros processos cognitivos são temas de estudos que objetivam uma

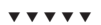

1 Artigo elaborado a partir da dissertação de L.M.C. ABURACHID, intitulada “Construção e validação de um teste de conhecimento tático declarativo: processos de percepção e tomada de decisão no tênis, mar". Universidade Federal de Minas Gerais, 2009. Apoio: Conselho Nacional de Desenvolvimento Científico e Tecnológico (Processo n 485989/2007-7) e a Coordenação de Aperfeiçoamento de Pessoal de Nível Superior, pela bolsa de mestrado.

2 Universidade Federal de Minas Gerais, Escola de Educação Física, Programa de Pós-Graduação em Ciências do Esporte. R. Padre Nóbrega, 290, Minas Brasil, 30730-230, Belo Horizonte, MG, Brasil. Correspondência para/Correspondence to: L.M.C. ABURACHID. E-mail: <lagusmar@ig.com.br>. 
melhor compreensão das suas funções e influências na ação tática de atletas, o que induz a uma verificação empírica desses construtos (McPherson \& French, 1991; Raab, 2003).

No levantamento bibliográfico realizado por Costa, Garganta, Fonseca e Botelho (2002) e complementado até o ano de 2008, assim como na revisão realizada para o desenvolvimento deste trabalho, observou-se que, nos últimos 10 anos, apenas os estudos de McPherson (1999), Domingues, Arroyo, Gallego, Gonzáles e Alvarez (2006) e McPherson e Kernodle (2007) tiveram como foco os processos de percepção e tomada de decisão no tênis. É importante destacar que esses trabalhos carecem de uma menção dos procedimentos adotados para sua validação e que foram aplicados por meio de entrevistas ou com uso de slides. Portanto, a situação de jogo que se apresentou aos atletas estava congelada, sem movimentação dos jogadores, o que tem sido questionado em trabalhos recentes (Féry \& Crognier, 2001; Tenenbaum, Sar-El \& Bar-Eli, 2000; Willians, Ward, Smeeton \& Allend, 2004). Tais trabalhos tratam das estratégias de percepção e antecipação no tênis, evidenciando que o treinamento desses processos cognitivos, através de simulação de vídeo, contribui para o desenvolvimento do treinamento tático em campo.

Neste estudo, imagens de jogos foram digitalizadas em computador, com o objetivo de tornar as ações do tênis apresentadas aos avaliados mais próximas da realidade ambiental. Assim, o sujeito tem a possibilidade de analisar toda a situação de jogo em sequência de tempo e espaço reais, para oportunamente escolher a melhor decisão a ser tomada na definição do ponto.

O objetivo do presente estudo foi, através do procedimento de coeficiente de validade de conteúdo, validar um número satisfatório de itens para a criação de um teste de Conhecimento Tático Declarativo (CTD) no Tênis (TCTD:Ts), que analise os processos de percepção e tomada de decisão que ocorrem no jogo. A validade de conteúdo é um tipo de validade interna realizada junto a outros procedimentos de validação, tais como, de critério e de construto que posteriormente são aplicadas para a aquisição os requisitos requeridos pela psicometria para se considerar um instrumento avaliativo. Com o intuito de aproximar a forma de apre-

262 sentação dos estímulos à maneira como eles ocorrem na realidade (Senior et al., 2000; Wrigth \& Jackson, 2007), foram coletadas cenas de jogos reais, em vídeo, para posterior processo de validação por peritos.

A busca pela validação das cenas de vídeo de tênis tem como principal desafio a criação de um teste que sirva de instrumento pedagógico para o esporte e a Educação Física. O teste visa contribuir para o desenvolvimento da capacidade tática dos praticantes da modalidade e, consequentemente, oferecer subsídios para orientar o processo de ensino-aprendizagem de tenistas.

No âmbito esportivo, a avaliação do CTD permite compreender os mecanismos por meio dos quais o jogador declara sua forma e nível de compreensão tática do jogo. Assim, explicando-se o que o tenista irá fazer de acordo com a lógica do jogo e a meta visada, é possível organizarem-se processos de ensino-aprendizado norteadores do treinamento.

Na literatura referente à solução de problemas e tomada de decisão no esporte, destaca-se o modelo elaborado por Greco (2006) sobre a relação entre conhecimento e memória e os diferentes processos cognitivos que envolvem o comportamento tático. As informações do organismo e do ambiente de jogo, que subsidiam a tomada de decisão e a concretização das ações, são processadas em três estruturas paralelas, que se inter-relacionam e se apoiam mutuamente. A estrutura perceptiva é constituída pela tríade dos processos de percepção-antecipação-atenção. O processamento da informação se constitui pelos processos de pensamento-inteligência, os quais se relacionam e interagem com a função primária de receber-codificar e de dar significado à informação, assim formatando o processo em paralelo da tomada de decisão tática. Quando a estrutura de recepção percebe como relevante um determinado sinal, o jogador prepara-se para tomar uma decisão através dos processos do pensamento, de modo que a colaboração paralela das três estruturas formata, em uma primeira instância (Roth, 1989; 1991), "o que fazer", e, em uma segunda instância, o "como fazer", concretizando a execução do gesto técnico necessário à solução do problema situacional. O modelo pendular da ação técnico-tática apresentado destaca a importância do ensino-aprendizado, bem como do treinamento dos processos cognitivos subjacentes à ação de forma situacional, isto é, em interação com o jogo (Figura 1). 


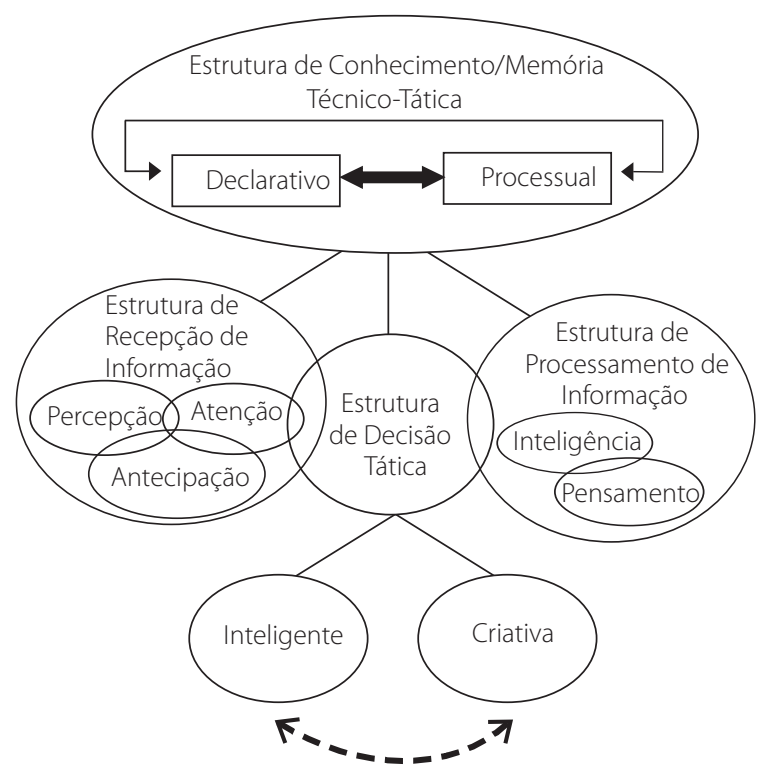

Figura 1. Modelo pendular da ação técnico-tática. Fonte: Greco (2006).

A seguir, a primeira seção deste trabalho detalha os métodos da pesquisa e os procedimentos realizados, a segunda apresenta os resultados e a terceira descreve a discussão e conclusões, destacando o valor dos itens validados para o uso de futuras pesquisas.

\section{Método}

\section{Procedimentos}

O estudo respeitou as normas estabelecidas pelo Conselho Nacional em Saúde, tendo sido aprovado pelo Comitê de Ética da Pesquisa da UFMG, com o parecer de n ETIC 23/08. Todos os participantes assinaram um termo de consentimento livre e esclarecido e tiveram preservada a identidade, tendo ainda a liberdade de abandonar a coleta a qualquer momento.

\section{Consolidação das cenas de vídeo}

As cenas de vídeo de jogos passaram por dois processos de peritagem, com o objetivo de se encontrarem altos valores de concordância, atestando fidedignidade. O resultado do nível de concordância interavaliadores pelo Kappa apresentou valores de 0,96. Após a aplicação da Técnica das metades e Prognóstico de
Sperman Brown, o nível de concordância intra-avaliador obteve um valor de 0,99. Os resultados acima confirmaram 190 cenas com valores de concordância elevados, excluindo-se apenas duas de um total de 192 cenas. Estas foram classificadas por ações técnico-táticas de golpe de fundo, approach, passada e voleio. Além disso, o teste de qui-quadrado $\left(\chi^{2}=56,51[p \leq 0,01]\right)$ foi aplicado para verificar a discrepância entre os resultados observados e esperados quanto aos valores de frequência absoluta nas diferentes ações técnico-táticas executadas pelos tenistas. Mantiveram-se 92 cenas de fundo de quadra, por serem ações mais frequentes, permanecendo no rol das possíveis de serem validadas (Aburachid \& Greco, 2010). Porém, em somente 43 delas as imagens apresentaram na parte inferior do vídeo o jogador que tomaria a decisão, aproximando o avaliado da semelhança de sua visão de jogo (Aburachid \& Greco, 2008).

Para a validação das cenas foram seguidos os procedimentos apresentados por Hernández-Nieto (2002), através da avaliação de um painel de juízes, conforme os critérios de clareza da imagem, pertinência prática e representatividade do item. O Coeficiente de Validade de Conteúdo (CVC) mediu o grau de concordância dos juízes em cada item, e também avaliou a validade de conteúdo, preenchendo a lacuna das propriedades métricas do Kappa, por exemplo, que avalia apenas a fidedignidade e a consistência. As avaliações dos peritos foram medidas por uma escala de Likert de 5 pontos, além de lhes permitir comentários livres em cada cena.

A seguir, apresentam-se os critérios para escolha das cenas:

Clareza de imagem: foi avaliada a imagem das cenas, levando-se em conta a população de tenistas que posteriormente iria responder a ela. Perguntou-se aos peritos: As imagens estão nítidas? É possível ver nitidamente a bola, os jogadores, a rede e as linhas da quadra nas cenas?

Pertinência prática: avaliou-se a relevância da cena como forma de representação das situações para tomada de decisão no jogo de tênis. Seguiu-se a pergunta: Você acredita que estas cenas representam situações adequadas para a tomada de decisão de um jogador em um jogo de tênis? 
Representatividade do item: avaliou-se o nível de demonstração dos processos cognitivos que se tinha interesse de avaliar através das cenas. Foi feita aos peritos a pergunta: Você acredita que esta cena permite a análise da tomada de decisão de um jogador e dos sinais relevantes que levam a ela?

Participaram deste estudo 5 voluntários no papel de juiz, número máximo recomendado na literatura para a validação de conteúdo. Seguindo as orientações de Balbinotti, Benetti e Terra (2006), o critério de seleção consistiu que eles não tivessem participado de nenhuma etapa da pesquisa, e, além disso, que tivessem experiência ativa de no mínimo dez anos na área. Ericsson, Charness, Feltovich e Hoffman (2006) conceituam o perito/juiz como indivíduo muito habilidoso, reconhecido em um campo específico, com um nível de perícia atingido através da prática deliberada. Para atingir esses critérios, os juízes que participaram eram treinadores de tênis que têm ou tiveram atletas juvenis com ranking entre os 10 primeiros colocados no circuito estadual ou nacional, ou que treinavam jogadores que competissem no cenário internacional.

Considerando que os procedimentos foram adotados conforme sugestão de Hernández-Nieto (2002), apenas foram aceitos valores de concordância $\geq 0,8$. Esse coeficiente possibilita avaliar o grau em que os itens representam os construtos através de ações intencionais. A folha entregue aos juízes para a realização da análise do CVC encontra-se no Anexo.

\section{Resultados}

Antes da exposição dos resultados, vale apresentar a fórmula específica utilizada para se estabelecer o coeficiente de validade de conteúdo (Quadro 1).

Os resultados do CVC foram designados separadamente na Tabela 1, para cada critério de validação de conteúdo. Os resultados demonstram que todos os critérios, em seus valores totais, obtiveram nota de partida de 0,83 de Coeficiente de Validade de Conteúdo Total do Critério (CVCt), inclusive no critério de pertinência prática (CVCt de 0,91), que afere se as cenas representam situações adequadas para a tomada de decisão em um jogo de tênis.

A representatividade do item, isto é, se as cenas permitem a análise da tomada de decisão e da percepção, alcançou CVCt de 0,98 em todos os itens, apresentando altos valores de validade de conteúdo.

Finalmente, no critério de clareza da imagem, que determina a nitidez desta em relação aos objetos presentes na situação de jogo - tais como bola, jogadores, rede e linhas da quadra -, apesar de ter como

Quadro 1. Fórmula do CVC e sua descrição.

\begin{tabular}{|c|c|c|c|}
\hline \multicolumn{4}{|c|}{ Fórmula do CVC } \\
\hline $1 M_{x}=\sum_{x} / J$ & ${ }^{2} \mathrm{CVCi}=\mathrm{M}_{x} / \mathrm{V}_{\text {máx }}$ & ${ }^{3} \mathrm{CVCC}=\mathrm{CVCi}-(1 / \mathrm{J})^{\mathrm{J}}$ & ${ }^{4} \mathrm{CVCt}=\operatorname{McvCi}-\mathrm{M}(1 / \mathrm{J})^{J}$ \\
\hline
\end{tabular}

Média de cada item: soma dos va- CVC do item: média do item pelo CVCC: CVC do item subtraído CVC total: média do CVC do item lores obtidos na escala de Likert valor máximo que o item pode al- da constante (Pei) da fórmula; subtraído da média da constante. (x) pelo número de juízes (J); cançar;

Nota: CVCi: coeficiente de validade de conteúdo de cada item; CVCc - coeficiente de validade de conteúdo de cada item subtraído da constante; Pei: constante da fórmula; CVCt: coeficiente de validade de conteúdo total do critério.

Tabela 1. Resultados do CVC para todos os critérios de validação.

\begin{tabular}{lccc}
\hline Critérios de validação & Itens com CVCi $<0,8$ & Itens com CVCi $\geq 0,8$ & Valor do CVCt \\
\hline Pertinência prática & 0 & 43 & 0,91 \\
Representatividade do item & 0 & 43 & 0,98 \\
Clareza da imagem & 4 & 39 & 0,89 \\
\hline
\end{tabular}

264 Nota: CVCi: coeficiente de validade de conteúdo de cada item; CVCt: coeficiente de validade de conteúdo total do critério. 
resultado um CVCt de 0,89 (5, 10, 12 e 35), apresentou quatro itens com Coeficiente de Validade de Conteúdo de cada Item Subtraído da Constante (CVCc) entre 0,71 e 0,79 . Esses valores, tidos como inaceitáveis pelos procedimentos de validação de Hernández-Nieto (2002), foram definitivamente descartados.

\section{Discussão}

De acordo com a avaliação dos juízes, as cenas foram nítidas em sua maioria, pertinentes e representativas em relação à proposta de avaliação da percepção e tomada de decisão no tênis. É interessante notar que os 4 itens descartados por não obterem valores de CVC $\geq 0,80$ não se ajustaram apenas ao critério de clareza de imagem, seguindo os valores de corte dos estudos de Balbinotti et al. (2006), Hernández-Nieto (2002) e Pasquali (1999; 2007). Esses resultados sugerem que a seleção de cenas foi pertinente com a busca por uma análise mais empírica.

Para se cobrir, pelo menos, grande parte da extensão semântica do construto, Pasquali (1999) recomenda que um teste deva conter um número final de 20 itens. Para isso, a psicometria assume que se deve iniciar o processo de validação com no mínimo o triplo de itens para posterior descarte, quando não são constituídos a partir de uma teoria. Quando constituídos por uma teoria, não é necessário iniciar com mais de 10\% além dos 20 itens a serem salvos. Nesse sentido este estudo apresentou um rigor desde a escolha e consolidação das cenas de vídeo, que se iniciaram com 197 imagens. Posteriormente, 43 cenas foram mantidas para processamento da validade de conteúdo e, finalmente, 39 cenas tornaram-se itens caracterizados como válidos do ponto de vista dos conteúdos, alcançando valores bem superiores aos recomendados pela literatura.

$\mathrm{O}$ alto número de cenas finais poderá ser utilizado em mais de uma versão do teste de conhecimento tático declarativo no tênis, já que, de acordo com o levantamento de Costa et al. (2002), um número mínimo de 6 e máximo de 13 itens são suficientes para avaliar esse tipo de construto.

\section{Considerações Finais}

Após a aplicação do CVC, foi confirmada a hipótese de validação de conteúdo, um número satisfatório de itens para a criação de um instrumento de avaliação do conhecimento tático no tênis, o que demonstra que esse método presta-se a validar não só questionários, mas também imagens em movimento.

Assim, esse tipo de validação vem contribuir como meio pedagógico, no maior conhecimento da modalidade e do nível de desenvolvimento do CTD dos tenistas, desde os primeiros anos de competição até o nível adulto/profissional. A aplicação do teste tornará possível planificar os processos de ensino-aprendizagem-treinamento do conhecimento tático. Avaliações temporais dentro dos períodos de treinamento permitirão adequada regulação do processo de formação.

\section{Referências}

Aburachid, L. M. C., \& Greco, P. J., (2008) Ações técnico-táticas em situação de definição no tênis. Revista Brasileira Ciência e Movimento, 16 (4), 1-16.

Aburachid, L. M. C., \& Greco, P. J. (2010). Processos de validação de um teste de conhecimento tático declarativo no tênis. Revista da Educação Física/UEM, 21 (4), 603-610.

Balbinotti, M. A., Benetti, C., \& Terra, P. R. S. (2006). Translation and validation of the Graham-Harvey survey for the brazilian context. International Journal of Managerial Finance, 3 (1), 26-48.

Costa, J. C., Garganta, J., Fonseca, A., \& Botelho, M. (2002). Inteligência e conhecimento específico em jovens futebolistas de diferentes níveis competitivos. Revista Portuguesa de Ciências do Desporto, 2 (4), 7-20.

Domínguez, A. M., Arroyo, M. P. M. , Gallego, D. I. , Gonzáles, L. G., \& Alvarez, F. V. (2006). Estudio del conocimiento declarativo en función de la experiencia y de la edad en jugadores jóvenes de voleibol. Cultura, Ciencia y Deporte, 3 (5), 73-80.

Ericsson, K. A., Charness, N., Feltovich, P. J., \& Hoffman, R. R. (2006). The Cambridge handebook of expertise and expert performance. New York: Cambridge University Press.

Féry, Y. A ., \& Crognier, L. (2001). On the tactical significance of game situations in anticipating ball trajectories in tennis. Research Quarterly for Exercise and Sport, 72 (2), 143-149.

Greco, P. J. (2006). Conhecimento tático-técnico: eixo pendular da ação tática (criativa) nos jogos esportivos coletivos. Revista Brasileira de Educação Física e Esporte, 20 (supl 5), 210-212.

Hernádez-Nieto, R. A. (2002). Contributions to statistical analysis. Mérida: Universidad de Los Andes.

McPherson, S. L. (1999). Tactical differences in problem representations and solutions in collegiate varsity and beginner female tennis players. Research Quarterly for Exercise and Sport, 70 (4), 369-384. 
McPherson, S. L., \& French, K. E. (1991). Changes in cognitive strategies and motor skill in tennis. Journal of Sport and Exercise Psychology, 13 (1), 26-41.

McPherson, S. L., \& Kernodle, M. (2007). Mapping two new points on the tennis expertise continuum: Tactical skills of adult advanced beginners and entry-level professionals during competition. Journal of Sports Sciences, 25 (8), 945-959.

Pasquali, L. (1999). Instrumentos psicológicos: manual prático de elaboração. Brasília: Prática Gráfica e Editora.

Pasquali, L. (2007). Validade dos testes psicológicos: será possível reencontrar o caminho? Psicologia: Teoria e Pesquisa, 23 (n. especial), 99-107.

Raab, M. (2003). Decision making in sport: influence of complexity on implicit and explicit learning. International Journal of Sport and Exercise Psychology, 1 (4), 406-433.

Roth, K. (1989). Taktik im sportspiel. Band 69. Bundes Institut für Sport und Sportwissenschaft. Schorndorf, Germany: Hofmann R. F.

Roth, K. (1991). Entscheidungsverhalten im sportspiel. Sportwissenschaft, 21 (3), 229-246.
Senior, C., Barnes, J., Giampietro, V., Simmons, A., Bullmore, E. T., Brammer, M., et al. (2000). The functional neuroanatomy of implicit-motion perception or 'representational momentum'. Current Biology, 10 (1), $16-22$

Tenenbaum, G., Sar-El, T., \& Bar-Eli, M. (2000). Anticipation of ball location in low and high-skill performers: a developmental perspective. Psychology of Sport and Exercise, 1 (2), 117-128.

Willians, A. M., Ward, P., Smeeton, N. J., \& Allend, D. (2004). Developing anticipation skills in tennis using on-court instruction: perception versus perception and action. Journal of Applied Sport Psychology, 16 (3), 350-360.

Wrigth, M. J., \& Jackson, R. C. (2007). Brain regions concerned with perceptual skills in tennis: an FMRI Study. International Journal of Psychophysiology, 63 (2), 214-220

Recebido em: 5/5/2009

Versão final reapresentada em: 27/8/2010

Aprovado em: 11/3/2011 
ANEXO

ORIENTAÇÕES PARA VALIDADE DE CONTEÚDO

Perito (a), dê notas para as cenas de definição em golpe de fundo no tênis em uma escala de Likert de 1 a 5 níveis, marcando um $\underline{X}$ sobre o número que significa o seu valor após a visualização da cena. Avalie, na sua opinião especializada, cada uma das cenas, levando em conta a clareza de imagem, pertinência prática e representatividade do item do teste e o nível de compreensão dos tenistas que competem com idade a partir de 12 anos.

Significado de cada conceito:

\begin{tabular}{ll}
\hline Clareza de Imagem & As imagens estão nítidas? É possível ver nitidamente a bola, os jogadores, a rede e as linhas da quadra nas \\
& cenas? \\
Pertinência prática & Você acredita que estas cenas representam situações adequadas para a tomada de decisão em um jogo de \\
& tênis? \\
Representatividade do item & Você acredita que esta cena permite a análise da tomada de decisão e dos sinais relevantes que levam a ela?
\end{tabular}

Use, para tanto, as escalas a seguir marcando com um $\underline{X}$ sua resposta EM CADA CENA:

\begin{tabular}{lll}
\hline & Escala de avaliação (Likert) & \\
\hline Clareza de Imagem & Pertinência prática & Representatividade do item \\
5 - muitíssima & 5 - muitíssima & 5 - muitíssima \\
4 - muita & 4 - muita & 4 - muita \\
3 - média & 3 - média & 3 - média \\
2 - pouca & 2 - pouca & 2 - pouca \\
1 - pouquíssima & 1 - pouquíssima & 1 - pouquíssima
\end{tabular}

\begin{tabular}{|c|c|c|c|}
\hline CENAS & Clareza de Imagem & Relevância Ecológica & Pentinência do Teste \\
\hline 1 & $1|2| 3|4| 5$ & $1|2| 3|4| 5$ & $1|2| 3|4| 5$ \\
\hline 2 & $1|2| 3|4| 5$ & $1|2| 3|4| 5$ & $1|2| 3|4| 5$ \\
\hline 3 & $1|2| 3|4| 5$ & $1|2| 3|4| 5$ & $1|2| 3|4| 5$ \\
\hline 4 & $1|2| 3|4| 5$ & $1|2| 3|4| 5$ & $1|2| 3|4| 5$ \\
\hline 5 & $1|2| 3|4| 5$ & $1|2| 3|4| 5$ & $1|2| 3|4| 5$ \\
\hline 6 & $1|2| 3|4| 5$ & $1|2| 3|4| 5$ & $1|2| 3|4| 5$ \\
\hline 7 & $1|2| 3|4| 5$ & $1|2| 3|4| 5$ & $1|2| 3|4| 5$ \\
\hline 8 & $1|2| 3|4| 5$ & $1|2| 3|4| 5$ & $1|2| 3|4| 5$ \\
\hline \multicolumn{4}{|c|}{........ até a cena 43.} \\
\hline
\end{tabular}


